\title{
Minimum Degree Distance of Five Cyclic Graphs
}

\author{
Nadia Khan ${ }^{1}$, Munazza Shamus ${ }^{1}$, Fauzia Ghulam Hussain ${ }^{1}$, Mansoor Iqbal ${ }^{2,}$ * \\ ${ }^{1}$ Department of Mathematics and Statistics, The University of Lahore, Lahore, Pakistan \\ ${ }^{2}$ National University of Comouter \& Emerging Sciences, Islamabad, Pakistan
}

Email address:

nadiakhan811@gmail.com (N. Khan), munazashms@gmail.com (M. Shamus), fauziahussain123@gmail.com (F. G. Hussain)

iqbalbcs@gmail.com (M. Iqbal)

${ }^{*}$ Corresponding author

\section{To cite this article:}

Nadia Khan, Munazza Shamus, Fauzia Ghulam Hussain, Mansoor Iqbal. Minimum Degree Distance of Five Cyclic Graphs. Pure and Applied Mathematics Journal. Vol. 10, No. 3, 2021, pp. 84-88. doi: 10.11648/j.pamj.20211003.13

Received: May 26, 2021; Accepted: July 28, 2021; Published: August 4, 2021

\begin{abstract}
Let $G$ be a connected graph with $\mathrm{n}$ vertices. Then the class of connected graphs having $\mathrm{n}$ vertices is denoted by $G_{n}$. The subclass of connected graphs with 5 cycles are denoted by $G_{n}{ }^{5}$. The classification of graph $G \in G_{n}{ }^{5}$ depends on the number of edges and the sum of the degrees of the vertices of the graph. Any graph in $G_{n}{ }^{5}$ contains five linearly independent cycles having at least $n+3$ edges and the sum of degrees of vertices of 5 -cyclic must be equal to twice of $n+4$. In this paper, minimum degree distance of class of five cyclic connected graph is investigated. To find minimum degree distance of a graph some transformations $T$ have been defined. These transformation have been applied on the graph $G \in G_{n}{ }^{5}$ in such a way that the resultant graph belongs to $G_{n}{ }^{5}$ and also degree distance of $T(G)$ is always must be less than $G$. For $\mathrm{n}=5$, the five 5 -cyclic graph has minimum degree distance 78 and the minimum degree distance of 5-cyclic graphs having six vertices is 124 . In case of $n$ greater than 6, a general formula for minimum degree distance is investigated. In this paper, we proved that the minimum degree distance of connected 5 cyclic graphs is $3 n^{2}+13 n-62$ by using transformations, for $n \geq 7$.
\end{abstract}

Keywords: Weiner Index, Graphical Sequence, Degree Distance, Five Cyclic Graphs

\section{Introduction}

For any graph $G \in G_{n}, d(x, y)$ represents the shortest distance between the vertices $x, y \in V(G)$ and the maximum of $d(x, y)$ for any vertices $x, y \in V(G)$ is defined to be the diameter of $G$, denoted by $\operatorname{giam}(G)$. A well-known topological index called the Wiener index [8] is of a graph $G$ is defined as

$$
\sum_{x \in V(G)} \sum_{x \in V(G)} d(x, y) .
$$

A new graph invariant other than Wiener index was introduced by Dobrynin and Kotchetova in [1] and Gutman [2] whose results are reinvestigated in [10] and is defined as for any given graph $G \in G_{n}$ the degree distance of a vertex $v \in V(G)$ is defined by

$$
D^{\prime}(v)=d(v) D(v)
$$

where $d(v)$ express the degree of a vertex $v$ and $D(v)=$ $\sum_{u, v \in V(G)} d(u, v)$. The degree distance of a graph $G$ is defined as:

$$
D^{\prime}(G)=\sum_{v \in V(G)} D^{\prime}(v)=\sum_{u, v \in V(G)} d(u, v)(d(x)+d(v)) .
$$

The two conjectures made by Dobrynin and Kochetova in [1] on the minimum and maximum values of the degree distance of a graph is discussed in [5]. In this paper, we determine all the extremal 5-cyclic graphs having the minimum degree distance.

In section 2, some known results are given for the proof of main result in this paper. In section 3, the characterization of extremal 5-cyclic graphs having minimum degree distance is discussed.

\section{Some Lemmas}

According to degree sequence trees have characterized by Moon [3] and Senior [4]. Connected unicyclic and bicyclic 
graphs has characterized according to degree sequence in [6]. Wei Zhu [7] has characterized connected tricyclic graphs via their degree sequence. N. Khan [9] has characterized connected four cyclic graphs according to degree sequence. The characterization of connected five cyclic graphs according to degree sequence is given in the following lemma.

Lemma 1. Let $n \geq 5$. The integers $n-1 \geq a_{1} \geq a_{2} \geq$ $\cdots \geq a_{n} \geq 1$, are the degrees of the vertices of a graph $G \in G_{n}^{5}$ are, iff

(i) The degrees sum of $n$-vertices of 5-cyclic graph $\sum_{i=1}^{n} a_{i}=2 n+8$

(ii) $d_{i} \geq 2$, for at least five indices.

Proof: $\Rightarrow$ Let $G \in G_{n}^{5}$. Then by the definition of 5-cyclic graph condition (i) and (ii) is satisfied. $W_{5}$ with an edge joining two alternate vertices of a cycle is the only 5-cyclic graph having the minimal order.

$\Leftarrow$ For $n=5$, we have $a_{1}+a_{2}+a_{3}+a_{4}+a_{5}=18$. If $a_{5} \geq 4$ then $a_{1}+a_{2}+a_{3}+a_{4}+a_{5} \geq 20$ a contradiction. So $a_{5}<4$. If $a_{5}=3$ then $a_{1}+a_{2}+a_{3}+a_{4}=15$, which implies that $a_{1}=a_{2}=a_{3}=4, a_{4}=3$. This degree sequence has a unique $G$ isomorphic to $W_{5}$ with an edge joining two alternate vertices of a cycle. If $a_{5}<3$, then $a_{5}=2$ and $a_{1}=a_{2}=a_{3}=a_{4}=4$ which is not a graphical sequence. Let $n \geq 6$ and assume that the result is true for all $k \leq n$.

Case 1. If $a_{n}>1$, then $a_{n}=2$. Otherwise, $a_{1}+a_{2}+\cdots+$ $a_{n} \geq 3 n>2 n+8$. For $a_{n}=2$ then for the equation $a_{1}+a_{2}+\cdots+a_{n-1}=2 n+6$, the following possibilities holds.

Subcase 1.1. If $a_{1}=10, a_{2}=a_{3}=\cdots=a_{n}=2$, this degree sequence represents a unique graph isomorphic to $G_{1}$ in figure 1.

Subcase 1.2. If $a_{1}=9, a_{2}=3, a_{3}=\cdots=a_{n}=2$, this degree sequence represents a unique graph isomorphic to $G_{2}$ or $G_{3}$ in figure 1 .

Subcase 1.3. If $a_{1}=8, a_{2}=a_{3}=3, a_{4}=\cdots=a_{n}=2$, this degree sequence represents a unique graph isomorphic to $G_{4}, G_{5}, G_{6}$ or $G_{7}$ in figure 1 .

Subcase 1.4. If $a_{1}=8, a_{2}=4, a_{3}=a_{4}=\cdots=a_{n}=2$, this degree sequence represents a unique graph is isomorphic to $G_{35}$ in figure 1 .

Subcase 1.5. If $a_{1}=7, a_{2}=a_{3}=a_{4}=3, a_{5}=\cdots=$ $a_{n}=2$, this degree sequence represents a unique graph isomorphic to $G_{8}, G_{9}$ or $G_{10}$ in figure 1 .

Subcase 1.6. If $a_{1}=7, a_{2}=5, a_{3}=a_{4}=a_{5}=\cdots=$ $a_{n}=2$, this degree sequence represents a unique graph isomorphic to $G_{6}$ or $G_{34}$ in figure 1 .

Subcase 1.7. If $a_{1}=7, a_{2}=4, a_{3}=3, a_{4}=\cdots=a_{n}=$ 2 , this degree sequence represents a unique graph isomorphic to $G_{33}$ in figure 1 .

Subcase 1.8. If $a_{1}=6, a_{2}=a_{3}=a_{4}=a_{5}=3, a_{6}=$ $\cdots=a_{n}=2$, this degree sequence represents a unique graph isomorphic to $G_{15}$ in figure 1 .

Subcase 1.9. If $a_{1}=6, a_{2}=a_{3}=4, a_{4}=a_{5}=a_{6}=$ $\cdots=a_{n}=2$, this degree sequence represents a unique graph isomorphic to $G_{11}, G_{12}, G_{13}$ or $G_{14}$ in figure 1 .
Subcase 1.10. If $a_{1}=a_{2}=6, a_{3}=a_{4}=3, a_{5}=a_{6}=$ $\cdots=a_{n}=2$, this degree sequence represents a unique graph isomorphic to $G_{16}$ in figure 1 .

Subcase 1.11. If $a_{1}=6, a_{2}=4, a_{3}=a_{4}=3, a_{5}=a_{6}=$ $\cdots=a_{n}=2$, this degree sequence represents a unique graph is isomorphic to $G_{32}$ in figure 1 .

Subcase 1.12. If $a_{1}=6, a_{2}=5, a_{3}=3, a_{4}=a_{5}=\cdots=$ $a_{n}=2$, this degree sequence represents a unique graph isomorphic to $G_{17}$ in figure 1 .

Subcase 1.13. If $a_{1}=5, a_{2}=a_{3}=a_{4}=a_{5}=a_{6}=3$, $a_{7}=\cdots=a_{n}=2$, this degree sequence represents a unique graph isomorphic to $G_{18}$ in figure 1 .

Subcase 1.14. If $a_{1}=4, a_{2}=a_{3}=a_{4}=a_{5}=a_{6}=a_{7}=$ $3, a_{8}=\cdots=a_{n}=2$, this degree sequence represents a unique graph isomorphic to $G_{31}$ in figure 1 .

Subcase 1.15. If $a_{1}=a_{2}=a_{3}=4, a_{4}=a_{5}=3, a_{6}=$ $a_{7}=\cdots=a_{n}=2$, this degree sequence represents a unique graph isomorphic to $G_{19}$ or $G_{30}$ in figure 1 .

Subcase 1.16. If $a_{1}=6, a_{2}=a_{3}=4, a_{4}=a_{5}=\cdots=$ $a_{n}=2$, this degree sequence represents a unique graph isomorphic to $G_{36}$ in figure 1 .

Subcase 1.17. If $a_{1}=a_{2}=5, a_{3}=4, a_{4}=a_{5}=\cdots=$ $a_{n}=2$, this degree sequence represents a unique graph isomorphic to $G_{37}$ in figure 1 .

Subcase 1.18. If $a_{1}=a_{2}=4, a_{3}=a_{4}=a_{5}=a_{6}=$ $3, a_{7}=\cdots=a_{n}=2$, this degree sequence represents a unique graph isomorphic to $G_{20}$ or $G_{21}$ in figure 1 .

Subcase 1.19. If $a_{1}=4, a_{2}=a_{3}=a_{4}=a_{5}=a_{6}=$ $a_{7}=3, a_{8}=\cdots=a_{n}=2$, this degree sequence represents a unique graph isomorphic to $G_{38}$ in figure 1 .

Subcase 1.20. If $a_{1}=a_{2}=a_{3}=a_{4}=4, a_{5}=a_{6}=$ $\cdots=a_{n}=2$, this degree sequence represents a unique graph isomorphic to $G_{22}, G_{23}$ or $G_{24}$ in figure 1 .

Subcase 1.21. If $a_{1}=a_{2}=5, a_{3}=a_{4}=3, a_{5}=a_{6}=$ $\cdots=a_{n}=2$, this degree sequence represents a unique graph isomorphic to $G_{25}$ in figure 1 .

Subcase 1.22. If $a_{1}=5, a_{2}=a_{3}=4, a_{4}=3, a_{5}=a_{6}=$ $\cdots=a_{n}=2$, this degree sequence represents a unique graph isomorphic to $G_{26}$ in figure 1 .

Subcase 1.23. If $a_{1}=a_{2}=a_{3}=a_{4}=a_{5}=a_{6}=a_{7}=$ $a_{8}=3, a_{9}=a_{10}=\cdots=a_{n}=2$, this degree sequence represents a unique graph isomorphic to $G_{27}, G_{28}$ or $G_{29}$ in figure 1.

Case 2. If $a_{n}=1$, we consider the following two subcases:

Subcase 2.1. If $a_{1}=n-1$, and for sufficiently large $n$, we have the following possibilities,

Subcase 2.1.1. If $a_{1}=n-1, a_{2}=\cdots=a_{11}=2, a_{12}=$ $\cdots=a_{n}=1$, this degree sequence represents a unique graph isomorphic to $H_{1}$ in figure 2 .

Subcase 2.1.2. If $a_{1}=n-1, a_{2}=a_{3}=3, a_{4}=\cdots=$ $a_{10}=2, a_{11}=\cdots=a_{n}=1$, this degree sequence represents a unique graph isomorphic to $H_{2}$ in figure 2 .

Subcase 2.1.3. If $a_{1}=n-1, a_{2}=a_{3}=a_{4}=a_{5}=$ $3, a_{6}=a_{7}=2, a_{8}=\cdots=a_{n}=1$, this degree sequence represents a unique graph isomorphic to $H_{3}$ in figure 2 .

Subcase 2.1.4. If $a_{1}=n-1, a_{2}=a_{3}=4, a_{4}=3, a_{5}=$ $a_{6}=2, d_{7}=\cdots=a_{n}=1$, this degree sequence represents a unique graph isomorphic to $H_{4}$ in figure 2 . 


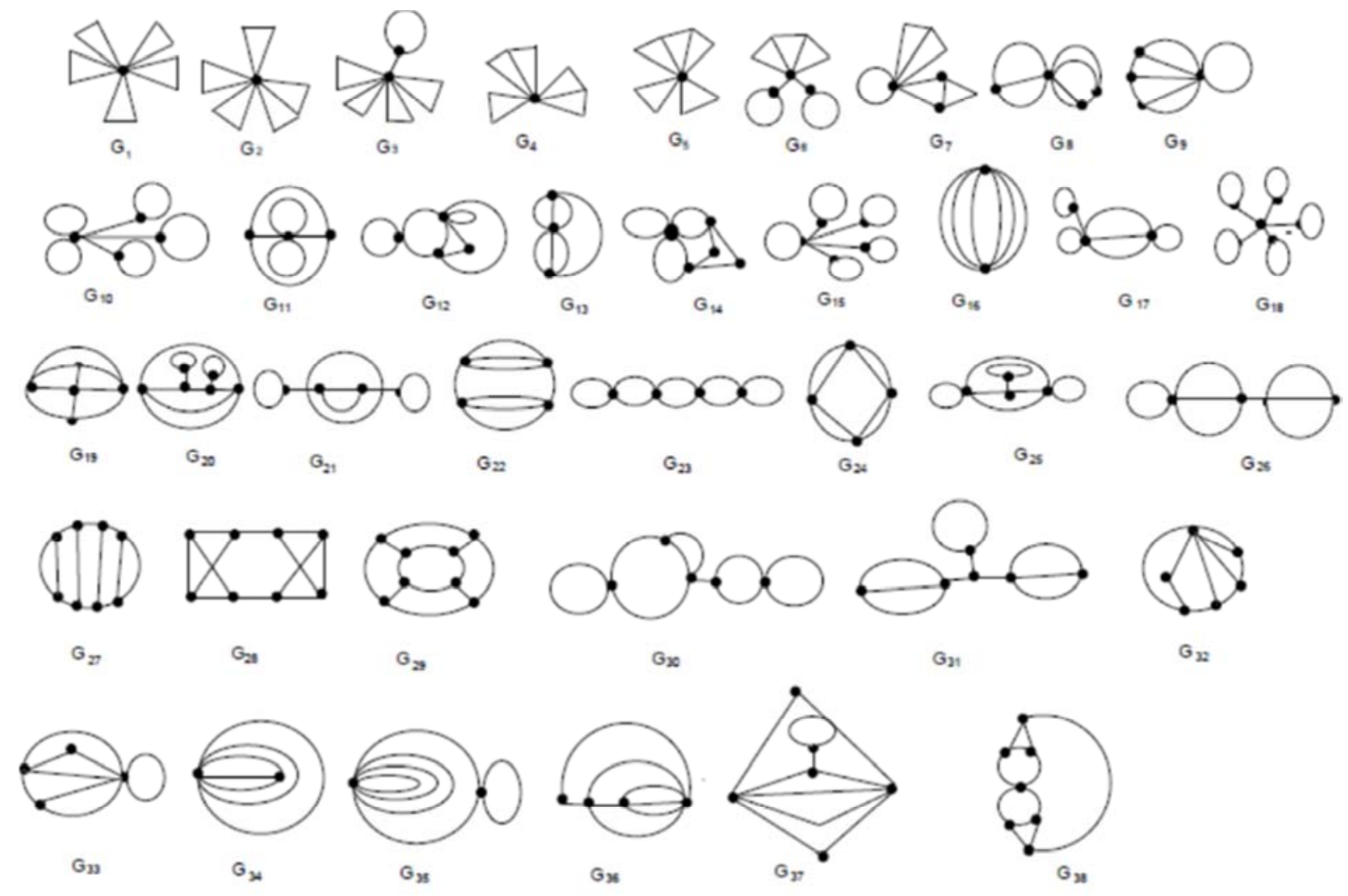

Figure 1. Five Cyclic Graphs with n-Vertices and $a_{n}=1$.

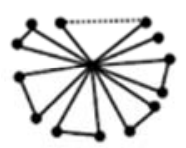

$\mathrm{H}_{1}$

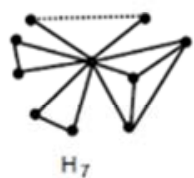

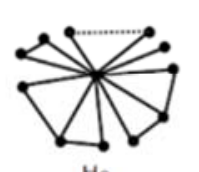

$\mathrm{H}_{2}$

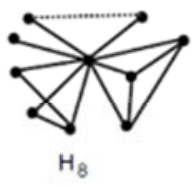

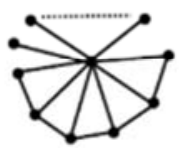

$\mathrm{H}_{3}$

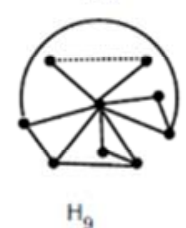

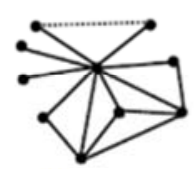

$\mathrm{H}_{4}$

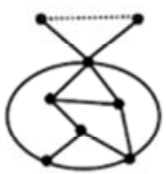

$\mathrm{H}_{10}$

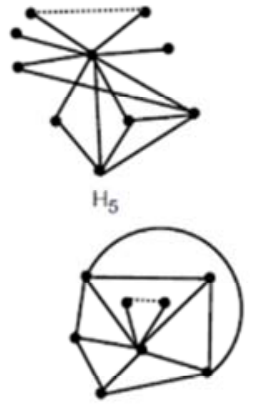

$\mathrm{H}_{11}$

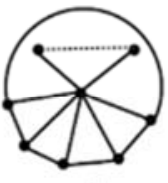

$\mathrm{H}_{6}$

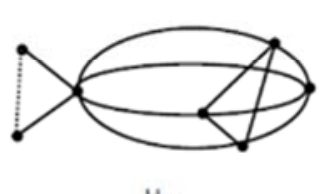

$\mathrm{H}_{12}$

Figure 2. Connected Five Cyclic Graphs with $n$-Vertices, $a_{1}=n-1$ and $a_{n}=1$.

Subcase 2.1.5. If $a_{1}=n-1, a_{2}=\cdots=a_{6}=3, a_{7}=$ $\cdots=a_{n}=1$, this degree sequence represents a unique graph isomorphic to $H_{6}$ in figure 2 .

Subcase 2.1.6. If $a_{1}=n-1, a_{2}=a_{3}=a_{4}=3, a_{5}=$ $a_{6}=a_{7}=a_{8}=2, a_{9}=\cdots=a_{n}=1$, this degree sequence represents a unique graph isomorphic to $H_{7}$ in figure 2 .

Subcase 2.1.7. If $a_{1}=n-1, a_{2}=a_{3}=a_{4}=a_{5}=$ $3, a_{6}=a_{7}=2, a_{8}=\cdots=a_{n}=1$, this degree sequence represents a unique graph isomorphic to $H_{8}$ or $H_{9}$ in figure 2 .

Subcase 2.1.8. If $a_{1}=n-1, a_{2}=a_{3}=4, a_{4}=a_{5}=3$, $a_{6}=\cdots=a_{n}=1$, this degree sequence represents a unique graph isomorphic to $H_{5}$ or $H_{11}$ in figure 2 .

Subcase 2.1.9. If $a_{1}=n-1, a_{2}=6, a_{3}=\cdots=a_{7}=2$, $a_{8}=\cdots=a_{n}=1$, this degree sequence represents a unique graph isomorphic to $H_{12}$ in figure 2 .

Subcase 2.2. Suppose $a_{1} \leq n-2$ and $a_{n}=1$. For each
$1 \leq k \leq n-1$ if $a_{k} \leq 2$ then $\sum_{k} a_{k} \leq 2 n-1$, which is a contradiction. There exists a maximum index $m, 1 \leq m \leq$ $n-1$, such that $a_{m} \geq 3$, and $a_{m+1} \leq 2$ and $a_{1} \geq a_{2} \geq \cdots \geq$ $a_{m-1} \geq a_{m} \geq \cdots \geq a_{n} \geq 1$. At minimum five members of the sequence $a_{1}, \cdots, a_{m-1}, a_{m}-1, \cdots, a_{n-1}$ are greater than 2, for which $a_{1} \leq n-2$ and $a_{1}+a_{2}+\cdots+a_{n}=$ $2(n-1)+8=2 n+6$. For this degree sequence there exist $G \in G_{n}^{5}$, by induction hypothesis. A vertex of degree $a_{m-1}$ is joined to a new vertex by an edge results a graph having four cycles with the degree sequence $a_{1} \geq a_{2} \geq \cdots \geq a_{n}=1$. Hence proof of Lemma 1 is complete.

If for any vertex $v \in V(G), d(v)=t$, then $D(v) \geq 2 n-$ $t-2$, and if for all $v \in V(G), d(v, y) \leq 2$ then $D(v)=$ $2 n-t-2$. Consequently, $D^{\prime}(G)=\sum_{v \in V(G)} d(v) D(v) \geq$ $\frac{1}{2} \sum_{t=1}^{n-1} t d_{t}(2 n-t-2)$, where $d_{t}$ denotes the number of 
vertices of degree $t, 1 \leq t \leq n-1$. By denoting as in [2], $F\left(x_{1}, x_{2}, \cdots, x_{n-1}\right)=\sum_{t=1}^{n-1} t d_{t}(2 n-t-2)$. We will find the minimum of $F\left(d_{1}, d_{2}, \cdots, d_{n-1}\right)$ over all-natural numbers $d_{1}, d_{2}, \cdots, d_{n-1} \geq 0$ satisfying the conditions in above lemma. We have the following corollary:

Corollary: Let $n \geq 5$. The integers $d_{1}, d_{2}, \cdots, d_{n-1} \geq 0$ are the multiplicities of the degrees of a graph $G \in G_{n}^{5}$ iff

(i) $\sum_{t=1}^{n-1} d_{t}=n$

(ii) $\sum_{t=1}^{n-1} t d_{t}=2 n+8$

(iii) $d_{1} \leq n-5$

Let the set of vectors of non-negative integers $d_{1}, d_{2}, \cdots, d_{n-1}$ be denoted by $\Delta$ satisfying the conditions (i)(iii) of corollary. Let us define transformations $T_{1}$ and $T_{2}$ $l \geq 2, m>0, l+m \leq n-2, x_{l} \geq 1, x_{m} \geq 1$, by

$T_{1}\left(d_{1}, \cdots, d_{n-1}\right)=\left(d_{1}^{\prime}, \cdots, d_{n-1}^{\prime}\right)=\left(d_{1}, \cdots, d_{l-1}+1, d_{l}-\right.$ $\left.1, \cdots, d_{l+m}-1, d_{l+m+1}+1, \cdots, d_{n-1}\right)$ and

$T_{2}\left(d_{1}, \cdots, d_{n-1}\right)=\left(d_{1}^{\prime}, \cdots, d_{n-1}^{\prime}\right)=\left(d_{1}, \cdots, d_{l-1}+1, d_{l}-\right.$ $\left.2, d_{l+1}+1, \cdots, d_{n-1}\right)$ we have $d_{i}=d_{i}^{\prime}$ for $i \neq\{l-1, l, l+$ $m, l+m+1\}$.

Lemma 2. Let $\left(d_{1}, \cdots, d_{n-1}\right) \in \Delta$ then

(a) $T_{1}\left(d_{1}, \cdots, d_{n-1}\right) \in \Delta$ if $l \neq 2$ and $x_{1} \neq n-5$, moreover $F\left(T_{1}\left(d_{1}, \cdots, d_{n-1}\right)\right)<F\left(d_{1}, \cdots, d_{n-1}\right)$

(b) $T_{2}\left(d_{1}, \cdots, d_{n-1}\right) \in \Delta$ if $l \neq 2$ and $x_{1} \neq n-5$, moreover

$F\left(T_{2}\left(d_{1}, \cdots, d_{n-1}\right)\right)<F\left(d_{1}, \cdots, d_{n-1}\right)$

Proof: (a). As $\sum_{t=1}^{n-1} d_{t}=\sum_{t=1}^{n-1} d_{t}^{\prime}$ and $\sum_{t=1}^{n-1} t d_{t}=$ $\sum_{t=1}^{n-1} t d_{t}^{\prime}=2 n+6$. If $\left(d_{1}, \cdots, d_{n-1}\right) \in \Delta, m=2$ and $n-$ 5 then $d_{1}^{\prime}>n-5$ a contradiction. Also $F\left(d_{1}, \cdots, d_{n-1}\right)-$ $F\left(T_{1}\left(d_{1}, \cdots, d_{n-1}\right)\right)=2 p+2>0$.

Similarly, (b) also hold.

\section{Main Result}

Theorem: Let $G \in G_{n}^{5}$,

(a) If $n=5$ then $\min D^{\prime}(G)=78$, where $G$ is a graph obtained from $W_{5}+$ an edge joining two vertices shown in figure 3 .

(b) If $n=6$ then $\min ^{\prime}(G)=124$ and all the extremal graphs are $A_{6}$ and $A_{8}$ in figure 4 .

(b) If $n \geq 7$ then $\min D^{\prime}(G)=3 n^{2}+13 n-62$, then all the extremal graphs are isomorphic to the graphs $E_{1}$ and $E_{2}$ in figure 5.

Proof. In order to find $\min F\left(d_{1}, \cdots, d_{n-1}\right)$ where $\left(x_{1}, \cdots, x_{n-1}\right) \in \Delta$.

Firstly, let $n=5$ then unique graph is given in Figure 3, and $D^{\prime}(G)=78$.

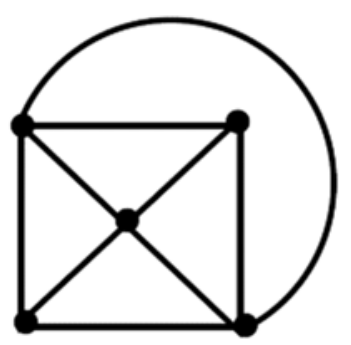

Figure 3. Five Cyclic Graph with five Vertices.

Secondly, let us consider $n=6$. Then all graphs $G \in G_{6}^{5}$ are $A_{i}$ for $1 \leq i \leq 8$ are shown in Figure 4. Here

$$
D^{\prime}\left(A_{1}\right)=D^{\prime}\left(A_{3}\right)=130, D^{\prime}\left(A_{2}\right)=132, D^{\prime}\left(A_{4}\right)=
$$

$D^{\prime}\left(A_{7}\right)=128, D^{\prime}\left(A_{5}\right)=126$ and $D^{\prime}\left(A_{6}\right)=D^{\prime}\left(A_{8}\right)=124$, respectively.

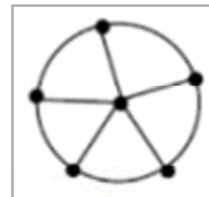

$A_{1}$

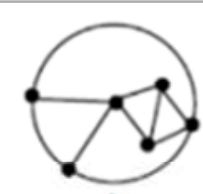

$\mathrm{A}_{2}$

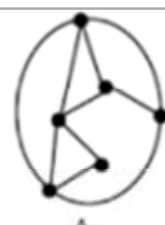

$\mathrm{A}_{3}$

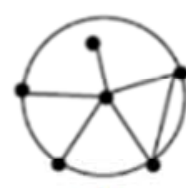

A

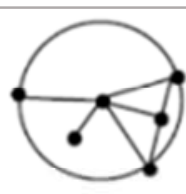

As

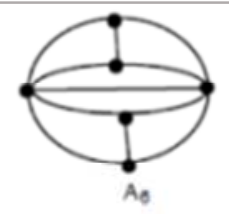

As

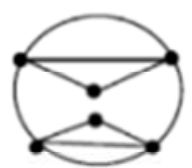

A 7

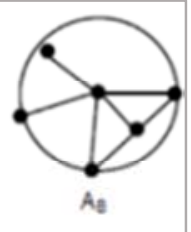

Figure 4. All Connected Six Cyclic Graphs with Six Vertices.

Finally, $n \geq 7$. If $d_{n-1} \geq 2$, take two distinct vertices $y_{1}, y_{2} \in V(G)$ such that $d\left(y_{1}\right)=d\left(y_{2}\right)=n-1$. As $n \geq 7$, by choosing at least seven different vertices which are distinct and adjacent to $y_{1}, y_{2}$ there exist at least six cycles, which is a contradiction. Therefore $d_{n-1} \ngtr 1$.

Now we determine the possible values of $d_{1}, d_{2}, \cdots, d_{n-2}$. If there exist $7<l, m<n-2$ such that $d_{l} \geq 1$ and $d_{m} \geq 1$ then a new vector $\left(d_{1}^{\prime}, \cdots, d_{n-1}^{\prime}\right) \in \Delta$ is obtained for which $\mathrm{F}\left(d_{1}^{\prime}, \cdots, d_{n-1}^{\prime}\right)<F\left(d_{1}, \cdots, d_{n-1}\right)$ by the application of the transformation $T_{1}$ for the position 1 and $\mathrm{m}$. we have for Similarly, if there exist $6<l, m<n-2$ such that $d_{l} \geq 2$ then a new degree sequence in $\Delta$ is obtained such that $\mathrm{F}\left(d_{1}^{\prime}, \cdots, d_{n-1}^{\prime}\right)<F\left(d_{1}, \cdots, d_{n-1}\right)$ by $T_{2}$. Now we consider two cases:

Case 1. Suppose that there exist distinct indices $\mathrm{i}, \mathrm{k}$ with $7<l, k<n-2$ such that $d_{l}=1$ and $d_{k}=0$. In this case, if $d_{6} \geq 1$ then a smaller value of $F$ is obtained by applying the $T_{1}$ for position 6 and 1 . Suppose that $d_{6}=0$. Since $d_{n-1} \in\{0,1\}$, two cases will be considered separately.

(a) In this case $d_{n-1}=d_{l}=1$, where $7<l$ and $d_{5}=0$. Pick different vertices $u, v, w, x, y, p, q \in V(G)$ such that $\mathrm{d}(\mathrm{u})=\mathrm{n}-1, \mathrm{~d}(\mathrm{v})=l \geq 7$ then $\mathrm{w}, \mathrm{x}, \mathrm{y}, \mathrm{p}, \mathrm{q}$ are all adjacent to $\mathrm{u}$ and $\mathrm{v}$ respectively. Also $\mathrm{u}$ and $\mathrm{v}$ are adjacent too, this leads to existence of six cycles which contradicts our hypothesis.

(b) If $d_{n-1}=0$ then $d_{5}=0$ and $d_{l}=1,(7<l, n-2)$ and $\Delta$ is characterized by the equations

$d_{1}+d_{2}+d_{3}+d_{4}=n-1$ and $d_{1}+2 d_{2}+3 d_{3}+4 d_{4}=$ $2 n+8-l$ which implies that $d_{2}+2 d_{3}=n+8-l$ by solving for $d_{2}$ and $d_{3}$ and then by applying the transformation for position 2 and 1 or 3 and 1 or 4 and 1, we obtain smaller value of $F$.

Case 2. Suppose that $d_{7}, \cdots, d_{n-1}=0$, hold and the degree sequence is

$\left(d_{1}, d_{2}, d_{3}, d_{4}, d_{5}, d_{6}, 0, \cdots, 0, d_{n}\right)$. As $d_{n-1} \in\{0,1\}$, so we have to analyze two cases: 
$\left(a^{\prime}\right)$. If $d x_{n-1}=0$, then $d_{2}+2 d_{3}+3 d_{4}+4 d_{5}+5 d_{6}=$ $n+8$. This equation does not hold. If all $d_{2}, d_{3}, d_{4}, d_{5}$ and $d_{6}$ are not greater than 2 , then $d_{2}+2 d_{3}+3 d_{4}+4 d_{5}+$ $5 d_{6} \leq 30$ which contradicts the hypothesis $n \geq 7$. If one of them is greater than two, then by using $T_{2}$ for the corresponding position, we obtain a smaller value of $F$.

$\left(b^{\prime}\right)$. If $d_{n-1}=1$, then $d_{2}+2 d_{3}+3 d_{4}+4 d_{5}+5 d_{6}=$ 10. If $d_{6} \geq 3$, then $d_{2}+2 d_{3}+3 d_{4}+4 d_{5}+5 d_{6} \geq 15$. So $d_{6} \leq 2$, if $d_{6}=2$ then $d_{2}+2 d_{3}+3 d_{4}+4 d_{5}=0$, which implies that $d_{2}=d_{3}=d_{4}=d_{5}=0$ and $d_{1}=n-3$ which is a contradiction as $d_{1} \leq n-5$. So $d_{6} \neq 2$. Thus either $d_{6}=0$ or $d_{6}=1$. If $d_{6}=1$, then $d_{2}+2 d_{3}+3 d_{4}+4 d_{5}=$ 5 , all the possible solutions are $d_{2}=1, d_{3}=2, d_{4}=$ $0, d_{5}=0$ or $d_{2}=3, d_{3}=1, d_{4}=0, d_{5}=0$ or $d_{2}=0$, $d_{3}=1, d_{4}=1, d_{5}=0$ or $d_{2}=1, d_{3}=0, d_{4}=0, d_{5}=$ 1. The third and fourth case should be removed as they contradicted corollary. From first and second case we obtain a degree sequence $(n-6,3,1,0,0,1,0, \cdots, 0,1)$ and (n $-5,2,0,1,0, \cdots, 0,1)$ which is not graphical.

Next consider if $d_{6}=0$, then then $d_{2}+2 d_{3}+3 d_{4}+$ $4 d_{5}=10$, all possible solution of $d_{2}, d_{3}, d_{4}$ and $d_{5}$ which follows the above corollary and construct the nine degree sequences which are graphical. The sequences are $(\mathrm{n}-$ $5,0,2,2,0, \cdots, 0,1),(n-6,0,5,0, \cdots, 0,1),(n-$

$6,2,2,0,1,0, \cdots, 0,1), \quad(\mathrm{n}-6,2,1,2,0, \cdots, 0,1),(\mathrm{n}-$ $7,4,1,0,1,0, \cdots, 0,1),(n-7,4,0,2,0, \cdots, 0,1),(n-$

$7,3,2,1,0, \cdots, 0,1), \quad(\mathrm{n}-8,5,1,1,0, \cdots, 0,1) \quad$ and $\quad(\mathrm{n}-$ $8,4,3,0, \cdots, 0,1)$. By applying transformations $T_{1}$ and $T_{2}$ these degree sequences are transformed to either the sequence $(n-5,0,2,2,0, \cdots, 0,1)$ or $(n-6,2,2,0,1,0, \cdots, 0,1)$ represented by graphs $E_{1}$ and $E_{2}$, respectively. And Hence it is proved

$F(\mathrm{n}-5,0,2,2,0, \cdots, 0,1)=F(\mathrm{n}-6,2,20,1,0, \cdots, 0,1)=$ $3 n^{2}+13 n-62$.

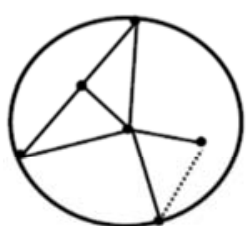

$E_{1}$

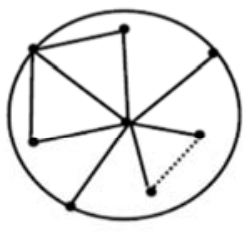

$\mathrm{E}_{2}$
Figure 5. Connected Six Cyclic Graphs with n-Vertices.

\section{Conclusion}

The minimum degree distance of connected five cyclic graphs $G_{n}^{5}$ is investigated. The graph contains five cycles if the sum of degrees of $n$ - vertices is equal to $n$ and sum of $\sum_{t=1}^{n} t d_{t}=2 \mathrm{n}+8$. A graph $G \in G_{n}^{5}$ if $G$ contains at least five vertices. The degree distance of $G \in G_{5}^{5}$ is $D^{\prime}(G)=78$ and $G \in G_{6}^{5}$ is $D^{\prime}(G)=124$. For $n \geq 7$, the minimum degree distance of $G \in G_{n}^{5}$ is $D^{\prime}(G)=3 n^{2}+13 n-62$.

\section{References}

[1] A. A. Dobrynin and A. A. Kochetova. "A Degree distance of a graph: A degree analogue of the Wiener index". J. Chem. Inform. Comput. Sci., 34 (1994), 1082-1086.

[2] I. Gutman. "Selected properties of the Schultz molecular topological index". J. Chem. Inform. Comput. Sci\}, 34 (1994), 1087-1089.

[3] J. W. Moon. "Counting Labelled Trees". Canadian Mathematical Monographs\}, Vol. 1, W. Clowes and Sons, London and Beccles, (1970).

[4] J. K. Senior. "Partitions and their representative graphs". Amer. J. Math., 73 (1951), 663-689.

[5] I. Tomescu. "Some extremal properties of the degree distance of a graph". Discrete Appl. Math., 98 (1999), 159-163.

[6] A. I. Tomescu. "Note on unicyclic and bicyclic graphs having minimum degree distance". Discrete Appl. Math., 156 (2008), 125-130.

[7] W. Zhu. "A note on tricyclic graphs with minimum degree distance". Discrete. Math. Algorithms and applications. Vol 3, No. 1 (2011) 25-32.

[8] H. Hosoya. "Topological index A newly proposed quantity characterizing the topological nature of structural isomers of saturated hydrocarbons". Bull. Chem. Soc Jpn., 4 (1971), 2332-2339.

[9] N. Khan, M. T. Rahim, Z. Raza. "A note on the degree distance of connected 4-cycle graph." Utilitas Mathematica 93 (2014), 109-116.

[10] M. Schocker. "On degree sequences of graphs with given cyclomatic number". Publ. Inst. Math, (N. S) 69 (2001), 3440. 